\title{
STUDY AND ANALYSIS OF IMPULSE NOISE REDUCTION FILTERS
}

\author{
Geoffrine Judith.M.C ${ }^{1}$ and N.Kumarasabapathy ${ }^{2}$ \\ ${ }^{1}$ EEE Department, Anna University of Technology Tirunelveli, Tirunelveli, India \\ geoffrine.judith@gmail.com \\ ${ }^{2}$ EEE Department, Anna University of Technology Tirunelveli, Tirunelveli, India \\ nkspaty@yahoo.co.in
}

\begin{abstract}
In this paper, a new Decision Based median filtering algorithm is presented for the removal of impulse noise from digital images. Here, we replace the impulse noise corrupted pixel by the median of the pixel scanned in four directions. The signal restoration scheme of this filter adapts to the varied impulse noise ratios while determining an appropriate signal restorer from a reliable neighbourhood. The experimental results of this filter applied on various images corrupted with almost all ratios of impulse noise favour the filter in terms of objectivity and subjectivity than many of the other prominent impulse noise filters.
\end{abstract}

\section{KEYWORDS}

Image restoration, impulse detection, impulse noise, median filter, RAMF

\section{INTRODUCTION}

The images corrupted by impulse noise are often occurred in practice. This type of noise may appear in digital images because of channel decoder damages, dyeing down of signal in communication links, communication subscriber's moving, video sensor's noises and other [1-3]. The impulse noise called salt and-pepper [4] causes white and black points appears in digital gray scale images, which chaotically scattered along image area. Applying of classic median filter for removal of such type of noise gives relatively good results, which are shown in restoring of brightness drops, objects edges and local peaks in noise corrupted images [4]. But analysis of different sources dedicated to median filtering shows, that the classic median filter has a set of disadvantages

- signal weakening (object's counters and edges are blurred in image);

- affecting to non corrupted ("good") image pixels.

Different modifications of median filter have been proposed to eliminate these disadvantages of median filtering. Now the switching scheme attracts a high interest of many researches [9-14]. This approach proves its efficiency for salt-and-pepper impulse noise removal from digital images. The switching scheme approach means splitting of noise removal procedure into two main stages $[10,14]$ :

1. Preliminarily detection of noise corrupted pixels of digital image.

2. Filtering of noise impulses which have been detected in first stage of processing using information about gathered image properties.

Described scheme with impulse detector is used in many advanced modifications of median filter. For example, in [15] authors purpose to use a detector based on fuzzy logic. And in [16] an

DOI : $10.5121 /$ sipij.2011.2107 
adaptive filter based on multilayer neural network [17] had offered for restoration of digital images. If the density of salt-and-pepper noise very high (more then 30\%), an algorithm described in $[11,25]$ is shown very efficient. This algorithm also developed using switching scheme. It uses sufficiently complex iterative procedures both at noise detecting stage and during filtering of corrupted image. This improves restoration of images even very extremely corrupted by impulse noise. In this work an improved algorithm proposed for impulse nose removal, which is able to restore images, corrupted by very intensive salt-and-pepper impulse noise, efficiently. The new algorithm has large performance in removal of impulse noise from digital images while having a relatively low complexity.

\section{MEDIAN FILTER}

Median filter, the most prominently used impulse noise removing filter, provides better removal of impulse noise from corrupted images by replacing the individual pixels of the image as the name suggests by the median value of the gray level of the pixels from a chosen neighbourhood. The median of a set of values is such that half of its values in the set are below the median value and half of them are above it and so is the most acceptable value than any other image statistics value for replacing the impulse corrupted pixel of a noisy image for if there is an impulse in the set chosen to determine the median it will strictly lie at the ends of the set and the chance of identifying an impulse as a median to replace the image pixel is very less. For a current image $f$ which is noisy, the median filter is a sliding square window of odd size that moves over the entire image, replaces individual pixel of the image by the median of all the pixels of the window. Let the pixel values within a square window $\mathrm{WxW}$ be represented spatially as

$$
\begin{gathered}
\Omega_{i}{ }^{w}=\left\{j=\left(j_{1}, j_{2}\right) / i_{1}-(w-1) / 2 \leq j_{1} \leq i_{1}+(w-1) / 2,\right. \\
\left.I_{2}-(w-1) / 2 \leq j_{2} \leq i_{2}+(w-1) / 2\right\}
\end{gathered}
$$

In correcting the impulse pixels, the median filter though shows satisfactory performance, makes numerous errors in on other vital aspects like the computational efficiency, mis- doing so. First of all, the median filter applies median of pixels from particular neighbourhood to all the pixels of the image irrespective of them being corrupted or not i.e., a pixel is identified noisy when there is no noise at all leading to noise suppression when the pixel is corrupted and signal suppression when the pixel is uncorrupted. The window size of the median filter also plays an important role in finding the most suitable value, larger and smaller windows lead to distortions when the impulse noise ratios are low and high respectively. Also the median value that is applied to the pixels of the noisy image is chosen from a neighbourhood without any constraints. This can be an inappropriate value i.e an impulse when the noise level is high, questioning the very aim of noise suppression filter.

These drawbacks put forward certain basic requirements to be satisfied by impulse noise filters. The noisy pixels alone are to be tracked (to be modified) by the most perfect value. Accordingly numerous algorithms got reported with distinct impulse tracking and correction phases, the Progressive pixel Switching Median Filter, the Adaptive Two-Pass Median Filter [20], the Rank Order based Adaptive Median Filter [RAMF] to restore highly corrupted images; the prime correction value being the median proved to be the most perfect. But these algorithms in the history of noise reduction could see good restoration only when the noise ratio is upto $40 \%$ beyond which [21]-[22] our new algorithm provide a better performance by addressing the limitations of former algorithms. 


\section{RAMF ( RANK-ORDER BASED ADAPTIVE MEDIAN FILTER)}

In RAMF, a pixel is retained if the median of a window around it is strictly between the minimum and the maximum value of the window and the pixel also is strictly between the minimum and the maximum value of the window; In case, the median of the window under consideration is strictly between the minimum and the maximum value of the window and the pixel does not lie strictly between the minimum and the maximum value of the window, then the pixel is replaced by the median value; otherwise, the size of the window is increased and the pixel will be replaced by the median of the increased size window, provided, this new median is strictly between the minimum and the maximum value of the window, otherwise, the window size is again increased up to some pre-fixed maximum level, beyond which the central pixel is left unchanged.

The strong advantage of RAMF is that it always ensures only those pixels which are not impulses are retained while ascertains that even in cases where the pixels are to be replaced by the median values, they are not impulses at all, making sure of preserving image details. This double edged advantage has kept this algorithm superior to several new algorithms reported subsequent to its publication.

It is pertinent to note that unlike RAMF which works very well for any type of image corrupted by impulse noise up to $50 \%$, they were good for some specific types of problems only. The limitation of this approach is that both non-impulsive pixels and pixels corrupted by impulse noises are considered for determining the median value of a window yielding a pseudo-median (here, 'pseudo' because of the fact that the median is not from among the reliable pixels) resulting in patches to occur in the images, especially when the impulse noise ratio increases beyond 3000 or more and when the salt and pepper impulse noises are unevenly distributed; i.e., when high frequency impulses are more than the low frequency impulses and vice versa.

Here, we often get only a noise as median, even though genuine non-impulsive pixels resides in the window under consideration. Increasing the window size in order to overcome the noise as median will affect the image fidelity. Again, even if we get a valid median, it need not be the actual median among the uncorrupted pixels except when the salt and pepper are equi-probable in the current window, which is a rare occurrence. Further the cases which could be disposed by just checking the validity of the central pixel are unnecessarily dragged to larger window computations.

\section{SWITCHING Median FiLter}

Switching Median impulse filter [23] identifies the noisy pixels by testing them for corruption with a more acceptable noise detector and replaced by a much valid intensity that can keep up the image fidelity to a large extent. The impulse detector is found from among the reliable pixels of window whose dimensions vary adaptively with the noise ratio which cannot cause the misdetection of signal as noise. The noisy pixels are replaced by a more reliable value obtained from a neighbourhood of the corrupted pixel.

Similar to other impulse detection algorithms [1]-[2] impulse detection is done by prior information on natural images, i.e., a noise-free image should be locally smoothly varying, and is separated by edges. The noise considered by this detection algorithm is only which means:

1) only a proportion of all the image pixels are corrupted while other pixels are noise-free and

2) a noise pixel takes either a very large value as a positive impulse or a very small value as a negative impulse. 
First, we create a 2D binary flag signal $b$ having the same size of the image $f$ to be filtered where bi and $f$ denote the flag value and signal (pixel) value at position $i=(i 1$ i2). We set bi $=1$ when the signal (pixel) value at spatial position $i$ is an impulse and bi $=0$ when the signal (pixel) value at $\mathrm{i}$ is non- impulsive.

Initially, we assume that all the pixels of the image to be filtered are impulses and set the 2D flag, $\mathrm{b}$ to 1 for all those pixels. In the detection process, for each pixel $\mathrm{f}$ we determine the minimum and maximum values of image $\mathrm{f}$ in the window $\mathrm{WD} \times \mathrm{WD}$ (where $\mathrm{WD}$ is an odd integer not smaller than 3 initialized to 3 ) centred at i. It is worth noting that salt is always a maximum and pepper a minimum in any given window.

Hence, if the centre pixel $\mathrm{f}$ strictly lies between minimum and maximum of a window, it is always likely to be a valid pixel which can be retained, and so we reset the flag at position i to 0 . If $f$ does not lie strictly between minimum and maximum values of the window chosen around it, i.e., when either $f=$ minimum or $f=$ maximum, we cannot be very sure that $f$ is an impulse, since chances are there that $f$ could be part of a smooth area without being an impulse.

If we use $\Omega_{\mathrm{i}}^{\mathrm{WD}}$ to represent the set of the pixel positions within a WD x WD window centred at I. By increasing the window size, we stand a good chance of getting another pixel which is smaller than $\mathrm{f}$ (=minimum) or greater than $\mathrm{f}$ (=maximum) as the case may be the increased window. It is worth noting that we also stand a very good chance of getting either a salt or pepper in the increased window.

In both cases, we can reset the flag at position i to 0 as $\mathrm{f}$ will strictly fall between the minimum and maximum of the increased window, we stop the detection process for $f$ and we move to the next pixel for noise detection. Otherwise we shall continue this exercise until we reach a prefixed maximum window size, $\mathrm{W}_{\text {Dma }}$ beyond which we conclude that the pixel is an impulse and move to the next pixel for checking. It is interesting to note that in this we need increase the window size, WD to a higher level whenever the impulse noise ratio goes to a very high level (i.e., more than $50 \%$ ) because in all probability, we may get both salt and pepper even in a smaller $3 \times 3$ window, enabling the decision making immediate.

\section{DECISION-BASED FILTER}

Decision Based Filter addresses the limitations of median filter, RAMF in which only median values are used for the replacement of the corrupted pixels. The new algorithm first detects the impulse noise in the image. The corrupted and uncorrupted pixels in the image are detected by checking the pixel element value against the maximum and minimum values in the window selected.

This new Decision Based Filter is used for the efficient restoration of highly impulse corrupted images. The impulse filtering technique identifies the noisy pixels by testing them for corruption with a more acceptable noise detector and replaces by a much valid intensity that can keep up the image fidelity to a large extent. The impulse detector is found from among the reliable pixels of the window to avoid the mis-detection of signals as noise. The noisy pixels are replaced by a more reliable value obtained from a closer neighbourhood of the corrupted pixel. First, we create a binary flag image $f$ having the same size of the image $X$ of size $m x n$ to be filtered where $f_{i}$ and $\mathrm{i} X$ denote the flag value and pixel value at position $\mathrm{i}=(\mathrm{i} 1, \mathrm{i} 2)$. We set $\mathrm{f}_{\mathrm{i}}=1$ when the pixel value at spatial position $i$ is an impulse and $f_{i}=0$ when the pixel value at $i$ is non-impulsive. Initially this flag image, $\mathrm{f}$ is set to ' 1 ' at all its spatial locations assuming that all the pixels of the image, $\mathrm{X}$ 
are corrupted. $\mathrm{K}$ is the number of iteration the image is scanned to calculate the replacement median value.The algorithm can be tracked through following steps:

\section{Algorithm:}

\section{Step 1:}

Process the first pass, $K=1$ starting from $i_{1}=1$ and $i_{2}=1$ and moving in the forward passion

\section{Step:2}

The local window size, $\mathrm{W}$ and the set of uncorrupted pixels, $\psi$ are initialized. i.e. $\mathrm{W}==3 ; \psi=\varphi$.

\section{Step 3:}

The set of pixel positions within a square window $\mathrm{W} \times \mathrm{W}$, centred at $\mathrm{i}=(\mathrm{i} 1, \mathrm{i} 2)$, is defined spatially by

$$
\begin{aligned}
\Omega_{\mathrm{i}}{ }^{\mathrm{w}}=\{ & \mathrm{j}=\left(\mathrm{j}_{1}, \mathrm{j}_{2}\right) / \mathrm{i}_{1}-(\mathrm{w}-1) / 2 \leq \mathrm{j}_{1} \leq \mathrm{i}_{1}+(\mathrm{w}-1) / 2, \\
& \left.\mathrm{i}_{2}-(\mathrm{w}-1) / 2 \leq \mathrm{j}_{2} \leq \mathrm{i}_{2}+(\mathrm{w}-1) / 2\right\}
\end{aligned}
$$

where $\mathrm{W}$ is an odd integer not less than 3 to indicate the size of the local neighbourhood under consideration. The phase begins by analyzing the pixel-wise characteristics of the corrupted image, $\mathrm{X}$ in the local neighbourhood, $\mathrm{WxW}$.

\section{Step 4:}

If $\mathrm{M}_{1}<\mathrm{X}_{\mathrm{i}}<\mathrm{M}_{2}$ then $\mathrm{X}_{\mathrm{i}}$ is declared uncorrupted and so the pixel is retained in the corresponding pixel position of the restored image, $\mathrm{U}$

i.e $\quad \mathrm{U}_{\mathrm{i}}^{\mathrm{K}}=\mathrm{X}_{\mathrm{i}}$

$\mathrm{M}_{1}=\operatorname{minimum}\left\{\mathrm{X}_{\mathrm{j}} / \mathrm{j} € \Omega_{\mathrm{i}}{ }^{\mathrm{W}}\right\}$

$\mathrm{M}_{2}=\operatorname{maximum}\left\{\mathrm{X}_{\mathrm{j}} / \mathrm{j} € \Omega_{\mathrm{i}}{ }^{\mathrm{w}}\right.$

Subsequently the flag image at position $\mathrm{i}$, fi is reset to ' 0 ' indicating a non-impulsive position. Now the algorithm is continued from Step 9.

\section{Step 5:}

The purity status of $X_{i}$ cannot be concluded corrupted when $X_{i}$ is found not to lie strictly in between $M_{1}$ and $M_{2}$. The uncorrupted pixels in the neighbourhood around $X_{i}$ are caught in the impulse free pixel set, $\psi$ i.e.

$$
\psi=\left\{\mathrm{X}_{\mathrm{j}} / \mathrm{j} € \Omega_{\mathrm{i}}{ }^{\mathrm{w}} \text { and } \mathrm{M}_{1}<\mathrm{X}_{\mathrm{j}}<\mathrm{M}_{2}\right\}
$$

\section{Step 6 :}

If the pixel is found corrupted and $\psi$ being non empty then $\mathrm{X}_{\mathrm{i}}$ is once again checked for it's purity by analyzing it's correlation with the uncorrupted pixels of the window, WxW for which $\psi$ $\min$ and $\psi_{\max }$ is determined in such a way that

$\psi_{\min }=\min (\psi) ; \psi_{\max }=\max (\psi)$;

by

The Minimum Deviation (MD) of $\mathrm{x}_{\mathrm{i}}$ with $\psi_{\min }$ and $\psi_{\max }$ is determined which is defined

$$
\mathrm{MD}=\operatorname{minimum}\left\{\psi_{\min }-\mathrm{X}_{\mathrm{i}}, \psi_{\max }-\mathrm{X}_{\mathrm{i}}\right\}
$$




\section{Step 7:}

Here if $\psi \neq \varphi$ and on MD $\leq$ Tc , the pixel $X_{\mathrm{i}}$ is declared uncorrupted and is maintained. Since it's correlation with the nearest uncorrupted signals is high. The corresponding flag image is also updated to ' 0 '. Now the algorithm is continued from Step 9.

\section{Step 8:}

Otherwise if $\psi \neq \varphi$ and MD > Tc then the pixel $\mathrm{X}_{\mathrm{i}}$ is replaced by the median of the pixels in $\psi$ which is the set of uncorrupted pixels in the nearest possible neighbourhood of $X_{i}$. Thus the restored output at the position $\mathrm{i}$ in the filtered image, $\mathrm{U}$ is obtained as follows.

$$
\mathrm{U}_{\mathrm{i}}^{\mathrm{K}}=\operatorname{median}(\psi)
$$

Subsequently the flag image $f_{i}$ is reset to ' 0 ' indicating a non-impulsive position since we replaced the corrupted pixel in the restored image. If $\psi$ is found empty at this stage, $\mathrm{X}_{\mathrm{i}}$ is replaced by the mean of four neighbours of the previously processed image as

$$
U k=\left\{\begin{array}{l}
\left(\frac{1}{4}\right) *(U k(i 1-1, i 2-1)+U k(i 1-1, i 2)+U k(i 1-1, i 2+1)+U k(i 1, i 2-1)) \text { if } K=1 \\
\left(\frac{1}{4}\right) *(U k(i 1+1, i 2+1)+U k(i 1+1, i 2)+U k(i 1+1, i 2-1)+U k(i 1, i 2+1)) \text { if } K=2 \\
\left(\frac{1}{4}\right) *(U k(i 1+1, i 2+1)+U k(i 1+1, i 2)+U k(i 1+1, i 2-1)+U k(i 1, i 2-1)) i f K=3 \\
\left(\frac{1}{4}\right) *(U k(i 1-1, i 2+1)+U k(i 1-1, i 2)+U k(i 1-1, i 2-1)+U k(i 1, i 2+1)) i f K=4
\end{array}\right.
$$

\section{Step 9:}

Move to next pixel for processing and repeat Step 1 through Step 8.

\section{Step 10:}

Increment $\mathrm{K}=\mathrm{K}+1$ and Move to Step 2 and process each pixel in the reverse passion from $\mathrm{i} 1=\mathrm{m}$ and $\mathrm{i} 2=\mathrm{n}$.

\section{Step 11:}

Increment $\mathrm{K}=\mathrm{K}+1$ and Move to Step 2 and process each pixel in the bottom left to top right passion from $\mathrm{i} 1=\mathrm{m}$ and $\mathrm{i} 2=1$ from $\mathrm{i} 1=\mathrm{m}$ and $\mathrm{i} 2=\mathrm{n}$.

\section{Step 12:}

Increment $\mathrm{K}=\mathrm{K}+1$ and Move to Step 2 and process each pixel in the reverse passion from $\mathrm{i} 1=1$ and $\mathrm{i} 2=\mathrm{n}$ from $\mathrm{i} 1=\mathrm{m}$ and $\mathrm{i} 2=\mathrm{n}$

\section{Step 13:}

Once the above four passes are over, we replace all the pixels in the final restored image $U$ by the median of the 4 individual pixel value in the above four scanning procedure 
The Decision Based Filter is featured to restore impulse corrupted digital images even in highly corrupted environments. The filtering scheme takes all steps to switch only the filtration of salt \& pepper impulse corrupted pixels by an initial estimation to identify the uncorrupted pixels and then by identifying the impulse corrupted pixels with an appropriate noise detecting threshold, TC. The threshold, TC used for detection of noisy pixels is a very small value which avoids misdetection of signals as noise. In this algorithm, as we have considered only the pixels that are not corrupted by salt \& pepper impulse from the smallest possible neighbourhood for determining the median values to replace the pixels corrupted with impulse noise, we always get the best possible restoration in comparison to most other variants of median filters including Median Filter, RAMF, Switching Median Filter enabling very good visual quality in the restored images even when the image is corrupted at very high level of salt \& pepper impulse noise to the tune of $95 \%$. The threshold TC is a very small value which avoids misdetection of signals as noises.

\section{EXPERIMENTAl RESUlts AND SimUlation ANALYSIS}

The performance of the Decision Based algorithm was tested over a wide variety of 8-bit gray scale images of 512×512 size against Median Filter, Rank-ordered Based Adaptive Median Filter (RAMF) and Switching Median filter. The new filter is found to perform quite well on images corrupted with impulse noise up to the level of 95\%. The Peak Signal to Noise Ratio (PSNR),Mean Square Error (MSE), Mean Absolute Error (MAE) and Computational Time (CT) are used to measure the objective dissimilarities between the filtered image and the original image. The Peak Signal to Noise Ratio (PSNR) is used to assess the signal preserving capability of the restoration algorithm

The peak signal to noise ratio (PSNR) [9] and Mean Absolute Error (MAE) has been used for numeric estimation of algorithm's efficiency in this work.

PSNR $=10 \log _{10}(255)^{2} / M S E$
Where, Mean Square Error $($ MSE $)=\frac{\sum_{i=1}^{m} \sum_{j=2}^{n}(z-A)^{2}}{m * n}$

And

Mean Absolute Error,

$$
\text { MAE }=\text { abs }\left\{\frac{\sum_{i=1}^{m} \sum_{j=1}^{n}(Z-A)}{m \circ n}\right\}
$$

where $\mathrm{Z}$ - Original image

A - Error image

$m$ - Number of columns in the image

$\mathrm{n}-$ Number of rows in the image

The PSNR values obtained using different filtering techniques on boat image at different levels of impulse noise are summarized in Table. 1. It can be noted that the new algorithm produces low MSE and MAE when compared to median Filter, RAMF, Switching Median. filters. It clearly shows the improved performance of the this filter towards impulse noise removal and signal preservation Fig.3 shows the restored outputs of the algorithms when the image is corrupted with varied salt and pepper impulse noise levels. Based on visual inspection, the 
Decision Based algorithm plays vital role in eliminating the impulse noise from the image while retaining the fine details.

In the case of high density noise, the performance of the standard methods is very poor in terms of noise suppression and detail preservation. This filter has proved its supremacy in preserving edges and fine details over the other prominent filters. Unlike the Decision Based filter, the comparative filters have not only flawed to retain the originality of edges but also have introduced non-existing edges, thereby making the image restoration unreliable; this aspect is a strong advantage of the new algorithm.

Even though the methods Median Filter, RAMF, Switching Median Filter requires short computational time, they failed to provide valuable restoration in terms of subjective and objective metrics. Those methods failed to preserve the fine details, such as the thin lines and sharp curves, in the restored images while the Decision Based filter preserves edge sharpness and reduces many artifacts in contrast to the other algorithms. For higher noise density the Decision Based Filter has higher PSNR value than when compared to Median Filter, RAMF, Switching Median Filter.

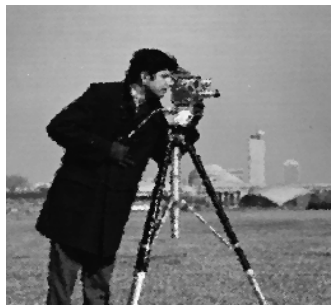

Fig.2 Original images used for analysis (a) Cameraman (b) Boats

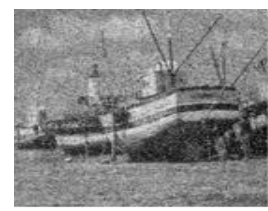

(a)

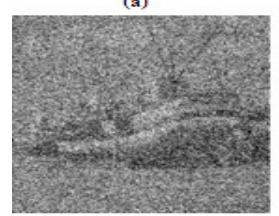

(f)

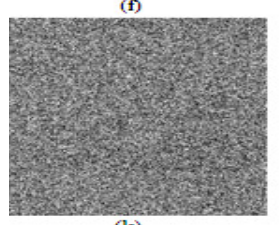

(k)

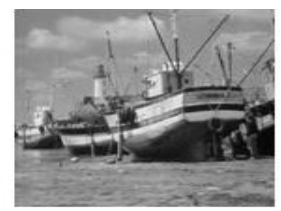

(b)

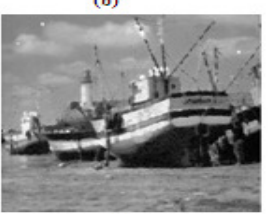

(g)

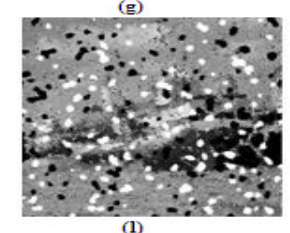

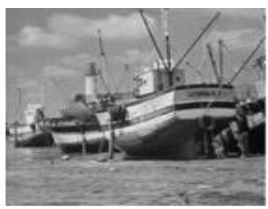

(c)

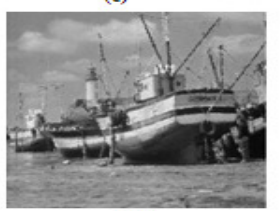

(h)

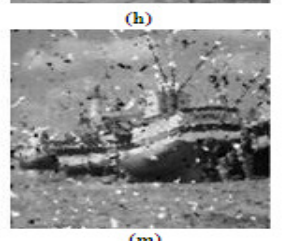

(m)

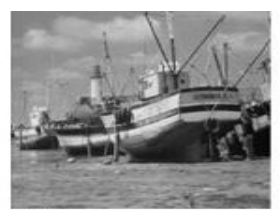

(d)

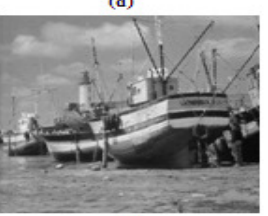

(i)

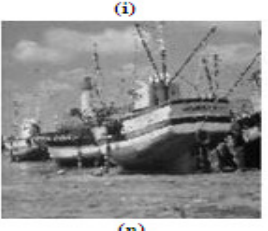

(n)

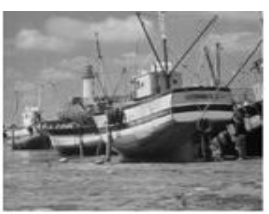

(e)

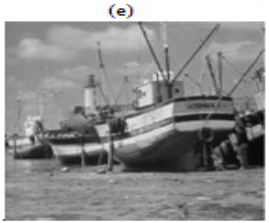

(j)

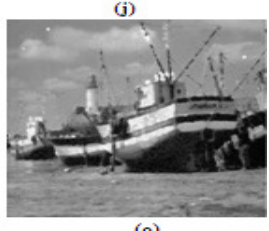

(o)

Fig.3. Shows images restored by Median Filter, RAMF, Switching Median Filter and Decision Based Filter respectively in each row against various levels of noise as follows:(a)Noise ratio at 20\%(f)Noise ratio at $60 \%(\mathrm{k})$ Noise ratio at $90 \%$ 


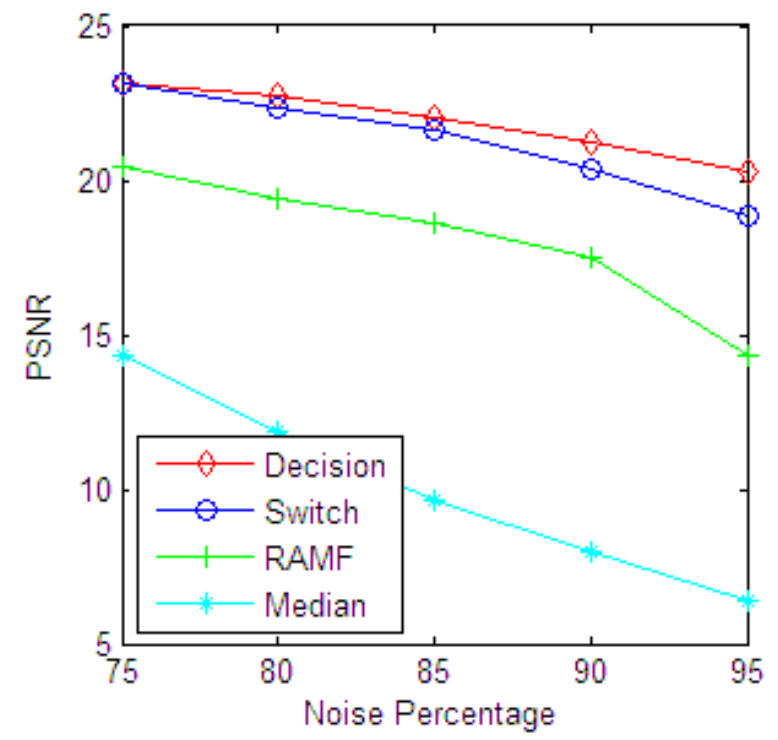

Fig.4 Performance Analysis of Decision Based Filter with Switching Median Filter , Median Filter and RAMF filters for Cameraman image.

\section{TABLE I}

\section{MAE, MSE, PSNR AND CT(SECONDS) AT DIFFERENT NOISE LEVELS FOR CAMERAMAN IMAGE}

\begin{tabular}{|c|c|c|c|c|c|c|c|c|c|c|c|c|c|c|c|c|}
\hline $\begin{array}{l}\text { NOISE } \\
\text { RATTO }\end{array}$ & \multicolumn{4}{|c|}{ MEDIAN } & \multicolumn{4}{|c|}{ RAMF } & \multicolumn{4}{|c|}{$\begin{array}{l}\text { SWITCHING MEDIAN } \\
\text { FILTER }\end{array}$} & \multicolumn{4}{|c|}{$\begin{array}{l}\text { DECISION BASED } \\
\text { FILTER }\end{array}$} \\
\hline & MAE & MSE & $\overline{\mathrm{PSNR}}$ & CT & MAE & MSE & PSNR & CT & MAE & MSSE & PSNR & $\mathrm{CT}$ & MAE & MSE & PSNR & CT \\
\hline 75 & 21.77 & 2412 & 14,31 & 1 & 9.2 & 588.9 & 20.4 & 2.9 & 6.6 & 313.07 & 23.17 & 5.58 & 6.8 & 314.16 & 23.16 & 25.85 \\
\hline 80 & 32.2 & 4267.4 & 11.83 & 1.1 & 10.69 & 748.2 & 19.4 & 3.68 & 7.5 & 378.4 & 22.35 & 6.5 & 7.4 & 348.3 & 22.7 & 20.2 \\
\hline 85 & 48.86 & 6997.9 & 9.68 & 1.1 & 12.19 & 891.98 & 18.63 & 5.1 & 8.5 & 451.1 & 21.6 & 7.62 & 8.4 & 407.34 & 22,03 & 23.2 \\
\hline 90 & 67.9 & 10075 & 8 & 1.2 & 14,73 & 1157 & 17.5 & 9 & 10.1 & 600.7 & 20.35 & 11.5 & 9.8 & 492.4 & 21.2 & 32.8 \\
\hline 95 & 97 & 15066 & 6.4 & 1.2 & 23.2 & 2403 & 14.3 & 25.8 & 12.5 & 843.4 & 18.87 & 18.5 & 12.2 & 609.7 & 20.3 & 43.76 \\
\hline
\end{tabular}

\section{CONCLUSION}

An efficient Decision Based algorithm is introduced for the restoration of images corrupted with almost all impulse noise levels. The filter is capable of producing recognizable, patches free outputs from images corrupted by higher levels of impulse noise. Experimental results show the feasibility of the new filter. This is affirmed by auditing the numerical measures like PSNR, MSE, MAE and visual observations. The filter provides maximum suppression of impulse noise while preserving fine details. 


\section{REFERENCES}

[1] Zhou Wang and David Zhang, "Progressive switching median filter for the Removal of impulse noise from highly corrupted images," IEEE Trans Circuits and Systems-II: Analog and Digital Signal Processing, vol. 46, no.1, Jan.1999.

[2] Hwang.H and Haddad.R.A, "Adaptive median filters: new algorithms and results," IEEE Trans. on Image Processing, vol.4, no.4, pp.499-502, 1995

[3] A. K. Jain, “Fundamentals of Digital Image Processing,” Englewood Cliffs,NJ: Prentice-Hall, 1989.

[4] A. C. Bovik, T. S. Huang, and D. C. Munson, "Edge-sensitive image restoration using orderconstrained least squares methods," IEEE Trans. Acoust., Speech, Signal Processing, vol. 33, pp. 1253-1263, Oct.1985.

[5]T. A. Nodes and N. C. Gallagher Jr., "The output distribution of median type filters," IEEE Trans. Commun., vol. COM-32, pp. 532-541, May 1984.

[6] A.C.Bovik., "Streaking in median filtered images," IEEE Trans. Acoust.,Speech,Signal Processing, n., vol. 35, pp. 493-503, Oct.1985.

[7.] D. A. F. Florencio and R.W. Shafer, "Decision-based median filter using local signal statistics," Proc. SPIE Symp.in Visual Communication and Image Processing, vol. 2308, Sept. 1994, pp. 268-275.

[8] T. Chen and H.Wu, "Adaptive impulse detection using center-weighted median filters," Signal Processing Lett., vol. 8, no. 1, pp. 1-3, Jan. 2001.

[9] R. Yang, L. Lin, M. Gabbouj, J.Astola, and Y. Neuvo,

"Optimal weighted median filters under structural constraints," IEEE Trans. Signal Processing, vol. 43, pp. 591-604, Mar. 1995.

[10] T. Song, M. Gabbouj, and Y. Neuvo, "Center weighted median filters:

some properties and applications in image processing," Signal Processing, vol. 35, no. 3, pp. 213-229, 1994.

[11] S. J. Ko and Y. H. Lee, "Center weighted median filters and their applications to image enhancement," IEEE Trans. Circuits Syst., vol. 38, pp.984-993, Sept.1991.

[13] T. Sun and Y. Neuvo, "Detail-preserving median based filters in image processing," Pattern Recognit. Lett., vol. 15, no. 4, pp. 341-347, Apr.1994.

[14]E. Abreu, M. Lightstone, S. K. Mitra, and K. Arakawa, "A new efficient approach for the removal of impulse noise from highly corrupted images,"IEEE Trans. Image Processing, vol. 5, pp. 1012-1025, 1996.

[15] H.-L. Eng and K.-K. Ma, "Noise adaptive soft-switching median filter," IEEE Trans. Image Processing, vol. 10, pp. 242-251, Feb. 2001.

[16] H. Lin and A. N. Wilson, "Median filter with adaptive length," IEEE Trans. Circuits Syst., vol. 35, pp. 675-690, June 1988.

[17] Shuqun Zhang and Mohammad A. Karim "A new impulse detector for switching median filters", IEEE Signal Processing Letters, Vol.9,No. 11, November 2002 
[18] Xiaoyin Xu, Eric L. Miller, Dongbin Chen and Mansoor Sarhadi "Adaptive two-pass rank order filter to remove impulse noise in highly corrupted images", IEEE Transactions on Image Processing, Vol. 13, No. 2, February 2004

[19] Denis K. Kuykin,Vladmir V.Khryashchev,Iilya V.Apalkov “ Improved Switching Median Filter",IEEE Transaction on Image Processing,2009

[20] Fabrizio Russo"New method for performance Evaluation of Grayscale Image Denoising Filters" IEEE Signal Processing Letters,Vol.17,No.5,May 2010

[21] Xiao-Guang Li "Impulse noise removal Using Grey Polynomial Model”IEEE transaction on Image Processing,2010.

[22] Kenny Kal Vin Toh," Noise Adaptive Fuzzy Switching Median Filter for Salt-and-pepper Noise Reduction”IEEE Signal Processing Letters, Vol 17,No 3,March 2010

[23] Krishnan Nallaperumal, Justin Varghese, S.Saudia, K.Arulmozhi, K.Velu,S.Annam, “Salt \& Pepper Impulse Noise Removal using Adaptive Switching Median Filter", IEEE Transactions on Image Processing, 2006

[24] Srinivasan.K.S and Ebenezer.D, "A new fast and efficient Decision - Based algorithm for removal of high - density impulse noises”, IEEE signal processing letters, vol.14, no.3, 2007.

[25] Madhu S. Nair, Revathy.K and Rao Tatavarti, "An improved decision - based algorithm for impulse noise removal", Congress on image and signal processing, 2008. 